\section{IN BRIEF}

\section{$\Rightarrow$ RHEUMATOID ARTHRITIS}

\section{Long-term response in RA apparent by 6 months}

An analysis of data from the British Society for Rheumatology Biologics Register for Rheumatoid Arthritis (BSRBR-RA) identified distinct patterns of response in patients with RA who began anti-TNF treatment between 2001 and 2013. The trajectories of treatment response were apparent within a few months of treatment initiation and reflected long-term outcomes. In a four-class model, most patients in the study followed a 'modest' (55.3\%) or 'substantial' (32.4\%) response trajectory, with $8.7 \%$ of the patients fitting the 'maximal' response trajectory (corresponding to sustained remission) and $3.6 \%$ the 'minimal' response trajectory.

ORIGINAL ARTICLE Hamann, P. D. H. et al. Early response to anti-TNF predicts long-term outcomes including sustained remission: an analysis of the BSRBR-RA. Rheumatology https://doi.org/10.1093/rheumatology/kez518 (2019)

\section{OSTEOARTHRITIS}

\section{Gut microbiome linked with OA knee pain}

Greater relative and absolute abundance of Streptococcus species in stool microbiota samples (a proxy for the gastrointestinal microbiome composition) was associated with more severe knee osteoarthritis (OA) and increased OA-related knee pain in a study of 1,427 patients from the population-based Rotterdam Study (RSIII) cohort.

The association was independent of smoking, alcohol intake and BMI. The association between Streptococcus spp. abundance and OA pain was replicated in an independent Dutch cohort $(n=867)$. Streptococcus spp. abundance was associated with MRI-detected inflammation in the knee joints, suggesting a possible explanation for its link with knee pain.

ORIGINAL ARTICLE Boer, C. G. et al. Intestinal microbiome composition and its relation to joint pain and inflammation. Nat. Commun. 10,4881 (2019)

\section{$\Rightarrow$ GOUT}

\section{LDHD mutation leads to hyperuricaemia and gout}

A mutation in the gene encoding lactate dehydrogenase $D$ $(L D H D)$ causes autosomal recessive gout with hyperuricaemia and underexcretion of uric acid, as evident in a study of a consanguineous Bedouin-Israeli family. Further investigation revealed that the mutation leads to excess blood D-lactate, which is excreted in exchange for reabsorbed uric acid, ultimately leading to hyperuricaemia and gout. Consistent with the clinical phenotype, injections of D-lactate into naive $\mathrm{C} 57 \mathrm{BL} / 6$ mice caused an increase in plasma uric acid concentrations.

ORIGINAL ARTICLE Drabkin, M. et al. Hyperuricemia and gout caused by missense mutation in d-lactate dehydrogenase.J. Clin. Invest. https://doi.org/10.1172/JCl129057 (2019)

\section{SPONDYLOARTHRITIS}

\section{Anti-TNF therapy reverses gut dysbiosis in AS}

Shotgun metagenome sequencing of DNA extracted from faecal samples collected from Han Chinese individuals reconfirmed the occurrence of bacterial gut dysbiosis in patients with ankylosing spondylitis (AS). The abundance of seven bacterial species differed between patients with AS $(n=127)$ and healthy individuals $(n=123)$. Treatment with a TNF inhibitor restored the relative abundance of these species in patients with AS to levels similar to those in healthy individuals.

ORIGINAL ARTICLE Yin, J. et al. Shotgun metagenomics reveals an enrichment of potentially cross-reactive bacterial epitopes in ankylosing spondylitis patients, as well as the effects of TNFi therapy upon microbiome composition. Ann. Rheum. Dis. https://doi.org/10.1136/annrheumdis-2019-215763 (2019)

\title{
First EULAR recommendations for Sjögren syndrome published
}

The care of patients with primary and secondary Sjögren syndrome is based primarily on symptomatic treatment, and has remained largely unchanged for decades. A project initiated in 2010 to develop the first evidence-based and consensusbased recommendations for the management of Sjögren syndrome has now culminated in the publication of three overarching principles and twelve specific recommendations.

The guidance synthesizes current thinking about Sjögren syndrome and the task force hopes that it will be widely applied in clinical practice. However, the publication also highlights the lack of diseasemodifying treatment options. "All randomized controlled trials (RCTs) performed to date with a reasonable number of patients (at least 100) have shown nonstatistically significant differences between the drug and the placebo arms," highlights Manuel RamosCasals, who led the task force. "There is no doubt that the results of RCTs so far are discouraging, especially taking into account that they were preceded by promising results in preliminary, small pilot studies."

The evidence base for the recommendations was limited to 9 RCTs, 18 prospective studies and 5 case-control studies. Thus, development of the published statements also incorporated the expertise of more than 100 professionals from 30 countries.

The group's first overarching recommendation is that Sjögren syndrome should be managed using a multidisciplinary approach, organised in collaboration with centres of expertise. The other two overarching principles recommend that first-line therapy for ocular and oral dryness should be topical therapies to relieve symptoms, and that systemic therapies can be considered for patients with active systemic disease.

The 12 specific recommendations are presented in a sequential approach, starting with treatment of the main symptoms of ocular and oral dryness, fatigue and pain, then addressing organ-specific systemic manifestations. They also emphasize tailoring treatment according to organ-specific severity, assessed using the EULAR Sjögren's syndrome disease activity index (ESSDAI).

Ramos-Casals notes that the therapeutic approach to ocular and oral dryness symptoms should be based on the results of objective diagnostic tests that measure the degree of glandular dysfunction, rather than on the patient's subjective assessment of symptom severity. The use of glucocorticoids, rituximab and immunosuppressive agents is also advised to be limited to use in patients with severe systemic disease, defined according to the ESSDAI score.

The task force also identified unmet needs and unanswered questions pertaining to Sjögren syndrome. "Research into this complex systemic autoimmune disease needs a multidisciplinary approach, the progressive and active involvement of patients, the use of big data and international cross-sectional studies," contends Ramos-Casals. Such efforts, together with data from a number of ongoing international studies such as the Sjögren Big Data Project, the European HARMONICSS study and the European NECESSITY study, could bring about substantial change in the management of Sjögren syndrome.

Sarah Onuora

ORIGINAL ARTICLE Ramos-Casals, M. et at. EULAR recommendations for the management of Sjögren's syndrome with topical and systemic therapies. Ann. Rheum. Dis. https://doi.org/ 10.1136/annrheumdis-2019-216114 (2019) 